\title{
Cerebral Microbleed Detection by Wavelet Entropy and Naive Bayes Classifier

\author{
Hai-nan WANG ${ }^{1}$ and Beatrice Gagnon ${ }^{1, a,{ }^{*}}$
}

${ }^{1}$ School of Computer Science and Technology, Nanjing Normal University, Nanjing, Jiangsu 210023, China

${ }^{1}$ State Key Laboratory of Digital Publishing Technology, Beijing 100871, China

${ }^{1}$ Division of Engineering Science, University of Toronto, Toronto, ON M5S2E4, Canada

ajsntwhn@hotmail.com, abgagnon_toronto@gmail.com

${ }^{*}$ Corresponding author

Keywords: Wavelet entropy, Cerebral microbleed, Naive Bayesian classifier.

\begin{abstract}
Aim) Current cerebral microbleed detection methods are too complicated, and difficult to train. (Method) We enrolled 10 subjects diagnosed as cerebral microbleed.Our method combined wavelet entropy and naive Bayes classifier. (Results) The simulation results over 10 times of 10 -fold cross validation showed that the average sensitivity, average specificity, and average accuracy of our method are $76.90 \pm 1.81 \%, 76.91 \pm 1.58 \%$, and $76.90 \pm 1.67 \%$, respectively. Our method can identify the CMB areas using only 1.41 seconds. (Conclusion) Our method is effective and rapid.
\end{abstract}

\section{Background}

The cerebral microbleed (CMB)[1] is a prodromal symptom of stroke. Nevertheless, detection of CMB by traditional structural magnetic resonance imaging (sMRI)[2-9] is difficult. The susceptibility weighted imaging (SWI)[10] is now attracting attention from both clinicians and technicians, since it can provide better accuracy than sMRI in detecting CMB.

In the last year, scholars have proposed many methods to detect CMB in SWI scanning. For example, Chen (2016) [11] suggested a novel leaky rectified linear unit (LReLU) classifier. Hou and Chen (2016) [12] proposed a four-layer deep neural network (DNN) method. Chen (2017) [13] extended the four-layer to a seven-layer neural network based on sparse autoencoders.

Nevertheless, above methods are too complicated. The training of either LReLU or the deep neural network are extremely time-consuming. Besides, the training results heavily rely on the initialization.

Hence, we suggested in this study to use a simple but efficient method, which is based on wavelet entropy and naive Bayesian classifier.Next Section 2 gives the materials and methods. Section 3 provides the experiments and results. Section 4 concludes the paper.

\section{Materials and Methods}

We enrolled 10 subjects diagnosed with cerebral microbleed (CMB). Their SWI images were reconstructed by Syngo MR B17 software. The size of the 3D volumetric image of each subject is $364 \times 448 \times 48$. An experienced physician is requested to label all the CMB areas, as shown in Figure 1. 


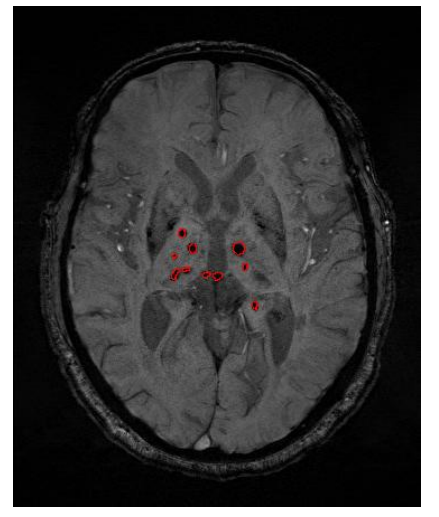

Figure 1 Samples of CMB areas

The sliding neighborhood processing technique is used to generate input and target samples from these 10 subjects. The sliding window size is chosen as $81 \times 81$. Finally, we generated $(364-80) *(448-80) * 48=5,016,576$ samples per subject. In all, we have $50,165,760$ samples for the total 10 subjects. Among them, 21965 samples are of CMB voxels, and the rest 50,143,795 samples are of healthy voxels.

To balance the dataset, we used random undersampling approach[14] to select 21981 healthy samples. Finally, our dataset contains 21965 CMB samples and 21981 healthy samples. Now this dataset is balanced.

The wavelet entropy[15-21] is successfully applied in many academic fields. In this study, it was employed to select important features from the sliding windows. We chose three-level decomposition. The wavelet family was selected as Haar wavelet.

The naive Bayes classifier [22] was used. It is a competitive method with state-of-the-art approaches, including support vector machines [23-26] and artificial neural networks[27-35].

\section{Results and Discussions}

The statistical results of our method are shown below in Table 1,

Table 2, and Table 3. Each row represents the result of one run, each column represents the result over each fold. The last column gives the total result over 10 folds.

Finally, the average sensitivity, average specificity, and average accuracy of our method are $76.90 \pm 1.81 \%, 76.91 \pm 1.58 \%$, and $76.90 \pm 1.67 \%$, respectively. This suggests the effectiveness of our method. For one slice image, our method can identify the CMB areas using only 1.41 seconds.

Table 1. Sensitivity of our method

\begin{tabular}{|l|l|l|l|l|l|l|l|l|l|l|l|}
\hline Run & F1 & F2 & F3 & F4 & F5 & F6 & F7 & F8 & F9 & F10 & Total \\
\hline R1 & 79.19 & 77.24 & 81.42 & 74.56 & 77.42 & 76.78 & 79.38 & 74.73 & 76.64 & 76.88 & 77.42 \\
\hline R2 & 74.95 & 78.37 & 75.73 & 79.33 & 80.70 & 77.78 & 78.06 & 80.43 & 78.15 & 77.06 & 78.06 \\
\hline R3 & 77.37 & 75.36 & 69.44 & 74.10 & 72.63 & 74.74 & 74.65 & 73.37 & 73.42 & 71.31 & 73.64 \\
\hline R4 & 74.13 & 75.23 & 75.74 & 75.60 & 73.54 & 78.78 & 74.01 & 75.24 & 75.56 & 79.19 & 75.70 \\
\hline R5 & 74.42 & 76.50 & 75.69 & 77.33 & 80.20 & 73.59 & 73.68 & 78.42 & 76.10 & 77.46 & 76.34 \\
\hline R6 & 74.37 & 78.28 & 80.51 & 79.47 & 80.33 & 77.00 & 84.07 & 82.42 & 80.29 & 78.38 & 79.51 \\
\hline R7 & 76.14 & 75.06 & 76.65 & 74.87 & 78.37 & 72.36 & 75.33 & 76.05 & 78.23 & 79.43 & 76.25 \\
\hline R8 & 74.41 & 78.10 & 75.47 & 72.59 & 77.38 & 76.05 & 70.87 & 76.06 & 77.19 & 76.15 & 75.42 \\
\hline R9 & 77.56 & 77.73 & 77.79 & 74.82 & 78.14 & 79.10 & 73.36 & 78.06 & 76.74 & 79.70 & 77.30 \\
\hline R10 & 80.52 & 80.70 & 77.32 & 78.61 & 82.29 & 80.70 & 78.01 & 78.73 & 78.33 & 77.82 & 79.30 \\
\hline
\end{tabular}


Table 2. Specificity of our method

\begin{tabular}{|l|l|l|l|l|l|l|l|l|l|l|l|}
\hline Run & F1 & F2 & F3 & F4 & F5 & F6 & F7 & F8 & F9 & F10 & Total \\
\hline R1 & 76.48 & 79.75 & 77.98 & 78.16 & 78.66 & 74.89 & 76.11 & 79.03 & 77.67 & 77.89 & 77.66 \\
\hline R2 & 77.12 & 79.25 & 78.90 & 78.21 & 76.25 & 77.53 & 79.85 & 77.48 & 76.62 & 78.43 & 77.96 \\
\hline R3 & 73.66 & 73.52 & 75.39 & 76.34 & 78.53 & 72.47 & 73.44 & 74.07 & 76.57 & 77.02 & 75.10 \\
\hline R4 & 75.34 & 76.89 & 75.11 & 75.22 & 76.34 & 72.20 & 73.34 & 75.07 & 76.07 & 76.16 & 75.17 \\
\hline R5 & 79.53 & 76.16 & 74.43 & 76.21 & 75.11 & 73.75 & 77.02 & 75.20 & 74.48 & 76.03 & 75.79 \\
\hline R6 & 78.62 & 78.34 & 77.84 & 81.03 & 76.07 & 79.31 & 79.03 & 83.44 & 80.48 & 79.03 & 79.32 \\
\hline R7 & 77.93 & 74.61 & 76.39 & 77.12 & 76.03 & 75.61 & 77.48 & 75.43 & 77.39 & 78.30 & 76.63 \\
\hline R8 & 77.17 & 76.43 & 76.93 & 74.34 & 76.11 & 76.11 & 74.43 & 72.66 & 73.38 & 75.48 & 75.31 \\
\hline R9 & 78.16 & 76.80 & 77.16 & 79.31 & 76.21 & 76.57 & 75.39 & 77.34 & 76.11 & 77.80 & 77.08 \\
\hline R10 & 75.89 & 82.26 & 80.80 & 77.13 & 78.43 & 80.35 & 78.80 & 78.53 & 77.80 & 80.98 & 79.10 \\
\hline
\end{tabular}

Table 3 Accuracy of our method

\begin{tabular}{|l|l|l|l|l|l|l|l|l|l|l|l|}
\hline Run & F1 & F2 & F3 & F4 & F5 & F6 & F7 & F8 & F9 & F10 & Total \\
\hline R1 & 77.83 & 78.50 & 79.70 & 76.36 & 78.04 & 75.83 & 77.75 & 76.88 & 77.16 & 77.38 & 77.54 \\
\hline R2 & 76.04 & 78.81 & 77.32 & 78.77 & 78.48 & 77.65 & 78.95 & 78.95 & 77.38 & 77.75 & 78.01 \\
\hline R3 & 75.51 & 74.44 & 72.42 & 75.22 & 75.58 & 73.61 & 74.04 & 73.72 & 74.99 & 74.17 & 74.37 \\
\hline R4 & 74.74 & 76.06 & 75.43 & 75.41 & 74.94 & 75.49 & 73.67 & 75.15 & 75.81 & 77.67 & 75.44 \\
\hline R5 & 76.97 & 76.33 & 75.06 & 76.77 & 77.66 & 73.67 & 75.35 & 76.81 & 75.29 & 76.75 & 76.07 \\
\hline R6 & 76.50 & 78.31 & 79.18 & 80.25 & 78.20 & 78.16 & 81.55 & 82.93 & 80.39 & 78.70 & 79.42 \\
\hline R7 & 77.04 & 74.84 & 76.52 & 76.00 & 77.20 & 73.99 & 76.41 & 75.74 & 77.81 & 78.86 & 76.44 \\
\hline R8 & 75.79 & 77.26 & 76.20 & 73.46 & 76.75 & 76.08 & 72.65 & 74.36 & 75.28 & 75.81 & 75.37 \\
\hline R9 & 77.86 & 77.26 & 77.47 & 77.06 & 77.17 & 77.83 & 74.37 & 77.70 & 76.43 & 78.75 & 77.19 \\
\hline R10 & 78.20 & 81.48 & 79.06 & 77.87 & 80.36 & 80.52 & 78.40 & 78.63 & 78.07 & 79.40 & 79.20 \\
\hline
\end{tabular}

\section{Conclusion and Future Research}

In this paper, we have proposed a novel CMB detection approach, based on wavelet entropy and naive Bayes classifier. The method achieved good results. In the future, we shall make tentative results on advanced feature extraction, such as wavelet Tsallis entropy [36-38]. Advanced classification methods shall be tested, including extreme learning machine [39] and linear regression classifier [40, 41].

\section{Acknowledgment}

This paper is supported by Research Innovation Program for College Graduates of Jiangsu Province (SJLX16_0284) and Opening Project of State Key Laboratory of Digital Publishing Technology.

\section{References}

[1] Wilson, D., A. Charidimou, G. Ambler, et al., Recurrent stroke risk and cerebral microbleed burden in ischemic stroke and TIA A meta-analysis. Neurology, 2016. 87(14): pp. 1501-1510

[2] Dong, Z., P. Phillips, G. Ji, et al., Exponential wavelet iterative shrinkage thresholding algorithm for compressed sensing magnetic resonance imaging. Information Sciences, 2015. 322: pp. 115-132

[3] Phillips, P., Z. Dong, and J. Yang, Pathological brain detection in magnetic resonance imaging scanning by wavelet entropy and hybridization of biogeography-based optimization and particle swarm optimization. Progress In 
Electromagnetics Research, 2015. 152: pp. 41-58

[4] Liu, A., Magnetic resonance brain image classification via stationary wavelet transform and generalized eigenvalue proximal support vector machine. Journal of Medical Imaging and Health Informatics, 2015. 5(7): pp. 1395-1403

[5] Yang, J., Preclinical diagnosis of magnetic resonance (MR) brain images via discrete wavelet packet transform with Tsallis entropy and generalized eigenvalue proximal support vector machine (GEPSVM). Entropy, 2015. 17(4): pp. 1795-1813

[6] Yu, D., H. Shui, L. Gen, et al., Exponential wavelet iterative shrinkage thresholding algorithm with random shift for compressed sensing magnetic resonance imaging. IEEJ Transactions on Electrical and Electronic Engineering, 2015. 10(1): pp. 116-117

[7] Dong, Z., Classification of Alzheimer disease based on structural magnetic resonance imaging by kernel support vector machine decision tree. Progress In Electromagnetics Research, 2014. 144: pp. 171-184

[8] Wu, L., Magnetic Resonance Brain Image Classification by an Improved Artificial Bee Colony Algorithm. Progress in Electromagnetics Research, 2011. 116: pp. $65-79$

[9] Wang, S. and L. Wu, A novel method for magnetic resonance brain image classification based on adaptive chaotic PSO. Progress in Electromagnetics Research, 2010. 109: pp. 325-343

[10] Bender, Y.Y.N., G. Diederichs, T.C. Walter, et al., Differentiation of Osteophytes and Disc Herniations in Spinal Radiculopathy Using Susceptibility-Weighted Magnetic Resonance Imaging. Investigative Radiology, 2017. 52(2): pp. 75-80

[11] Chen, Y. Voxelwise detection of cerebral microbleed in CADASIL patients by leaky rectified linear unit and early stopping: A class-imbalanced susceptibility-weighted imaging data study. Multimedia Tools and Applications, 2016, DOI: $10.1007 / \mathrm{s} 11042-017-4383-9$ (Online).

[12] Hou, X.-X. and H. Chen. Sparse Autoencoder based deep neural network for voxelwise detection of cerebral microbleed. in 22nd International Conference on Parallel and Distributed Systems. 2016. Wuhan, China: IEEE. pp. 1229-1232

[13] Chen, H. Seven-layer deep neural network based on sparse autoencoder for voxelwise detection of cerebral microbleed. Multimedia Tools and Applications, 2017, DOI: $10.1007 / \mathrm{s} 11042-017-4554-8$ (Online).

[14] Maglietta, R., N. Amoroso, M. Boccardi, et al., Automated hippocampal segmentation in 3D MRI using random undersampling with boosting algorithm. Pattern Analysis And Applications, 2016. 19(2): pp. 579-591

[15] Wang, S.-H. Single slice based detection for Alzheimer's disease via wavelet entropy and multilayer perceptron trained by biogeography-based optimization. Multimedia Tools and Applications, 2016, DOI: 10.1007/s11042-016-4222-4 (Online).

[16] Lu, H.M., Facial Emotion Recognition Based on Biorthogonal Wavelet Entropy, Fuzzy Support Vector Machine, and Stratified Cross Validation. IEEE Access, 2016. 4: pp. 8375-8385

[17] Nayak, D.R. Detection of unilateral hearing loss by Stationary Wavelet Entropy. CNS \& Neurological Disorders - Drug Targets, 2017. 16, DOI: 10.2174/1871527315666161026115046 (Online).

[18] Gorriz, J.M. and J. Ramírez, Wavelet entropy and directed acyclic graph support vector machine for detection of patients with unilateral hearing loss in MRI scanning. Frontiers in Computational Neuroscience, 2016. 10, Article ID: 160

[19]Zhou, X.-X., Comparison of machine learning methods for stationary wavelet 
entropy-based multiple sclerosis detection: decision tree, k-nearest neighbors, and support vector machine. Simulation, 2016. 92(9): pp. 861-871

[20] Zhou, X.X. and G.S. Zhang, Detection of abnormal MR brains based on wavelet entropy and feature selection. IEEJ Transactions on Electrical and Electronic Engineering, 2016. 11(3): pp. 364-373

[21] Sun, P., Pathological brain detection based on wavelet entropy and Hu moment invariants. Bio-Medical Materials and Engineering, 2015. 26(s1): pp. 1283-1290

[22] Ashfaq, T. and K. Khurshid, Classification of Hand Gestures Using Gabor Filter with Bayesian and Naive Bayes Classifier. International Journal Of Advanced Computer Science And Applications, 2016. 7(3): pp. 276-279

[23] Yang, J., Identification of green, Oolong and black teas in China via wavelet packet entropy and fuzzy support vector machine. Entropy, 2015. 17(10): pp. 6663-6682

[24] Ji, G., An MR brain images classifier system via particle swarm optimization and kernel support vector machine. The Scientific World Journal, 2013, Article ID: 130134

[25] Wu, L., Classification of fruits using computer vision and a multiclass support vector machine. Sensors, 2012. 12(9): pp. 12489-12505

[26] Wu, L., An MR brain images classifier via principal component analysis and kernel support vector machine. Progress In Electromagnetics Research, 2012. 130: pp. 369-388

[27] Zhang, Y., L. Wu, and S. Wang, Bacterial foraging optimization based neural network for short-term load forecasting. Journal of Computational Information Systems, 2010. 6(7): pp. 2099-2105

[28] Wu, L., Crop Classification by forward neural network with adaptive chaotic particle swarm optimization. Sensors, 2011. 11(5): pp. 4721-4743

[29] Lu, Z., A Pathological Brain Detection System Based on Radial Basis Function Neural Network. Journal of Medical Imaging and Health Informatics, 2016. 6(5): pp. 1218-1222

[30] Wu, J., Fruit classification by biogeography-based optimization and feedforward neural network. Expert Systems, 2016. 33(3): pp. 239-253

[31] Zhang, Y., Stock market prediction of S\&P 500 via combination of improved BCO approach and BP neural network. Expert systems with applications, 2009. 36(5): pp. 8849-8854

[32] Chen, M., Y. Li, and L. Han, Detection of dendritic spines using wavelet-based conditional symmetric analysis and regularized morphological shared-weight neural networks. Computational and Mathematical Methods in Medicine, 2015, Article ID: 454076

[33] Zhang, Y.D., L.N. Wu, G. Wei, et al., A novel algorithm for all pairs shortest path problem based on matrix multiplication and pulse coupled neural network. Digital Signal Processing, 2011. 21(4): pp. 517-521

[34] Feng, C., Feed-forward neural network optimized by hybridization of PSO and ABC for abnormal brain detection. International Journal of Imaging Systems and Technology, 2015. 25(2): pp. 153-164

[35] Ji, G., Fruit classification using computer vision and feedforward neural network. Journal of Food Engineering, 2014. 143: pp. 167-177

[36] Liu, G., Pathological brain detection in MRI scanning by wavelet packet Tsallis entropy and fuzzy support vector machine. SpringerPlus, 2015. 4(1), Article ID: 716

[37] Chen, P. and S. Du, Pathological Brain Detection via Wavelet Packet Tsallis Entropy and Real-Coded Biogeography-based Optimization. Fundamenta 
Informaticae, 2017. 151(1-4): pp. 275-291

[38] Wu, L., Optimal multi-level Thresholding based on Maximum Tsallis Entropy via an Artificial Bee Colony Approach. Entropy, 2011. 13(4): pp. 841-859

[39] Lu, S. and X. Qiu, A Pathological Brain Detection System based on Extreme Learning Machine Optimized by Bat Algorithm. CNS \& Neurological Disorders Drug Targets, 2017. 16(1): pp. 23-29

[40] Du, S., Alzheimer's Disease Detection by Pseudo Zernike Moment and Linear Regression Classification. CNS \& Neurological Disorders - Drug Targets, 2017. 16(1): pp. 11-15

[41] Chen, Y., A Feature-Free 30-Disease Pathological Brain Detection System by Linear Regression Classifier. CNS \& Neurological Disorders - Drug Targets, 2017. 16(1): pp. 5-10 